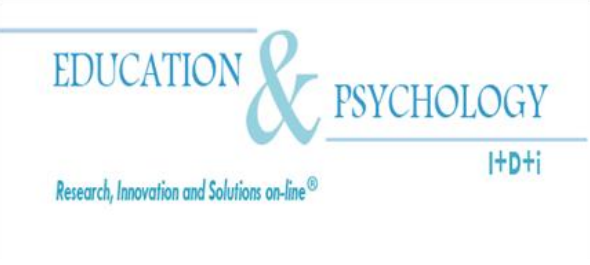

\title{
La indisciplina social e instruccional en adolescentes y universitarios: la influencia del género
}

\section{$M^{a}$ del Mar Badia Martín ${ }^{1}$, Ramon Cladellas Pros ${ }^{1}$, Concepció Gotzens Busquets ${ }^{2}$, Mercè Clariana Muntada ${ }^{1}$}

\author{
${ }^{1}$ Departamento de Psicología Básica, Evolutiva y de la Educación \\ Universitat Autònoma de Barcelona \\ ${ }^{2-}$ Departamento de Pedagogía Aplicada y Psicología de la educación. \\ Universitat Islas Baleares
}

\section{España}

Correspondencia: M.del Mar Badia Martín. Departamento de Psicología Básica, Evolutiva y de la Educación. Campus de Bellaterra Edificio B.Universitat Autònoma de Barcelona (España). E-mail: mar.badia@uab.es

(C) Education \& Psychology I+D+i and Editorial EOS (Spain) 


\section{Resumen}

Introducción. Son diversos los estudios sobre las diferencias de género en la escuela. Pero son escasas las investigaciones que hayan tenido en cuenta la variable género en el estudio de los comportamientos disruptivos convencionales e instruccionales. En este trabajo pretendemos hacer un acercamiento al tema.

Método. Esta investigación es un estudio cuantitativo empírico-analítico, que compara los datos obtenidos a partir de la aplicación de un cuestionario a 456 sujetos, alumnos de bachillerato de centros públicos y privados de Barcelona y Madrid, y de la Universidad Autónoma de Barcelona (España). Para este estudio se desarrolló una herramienta que consistía en una diana formada por círculos concéntricos y un listado de comportamientos disruptivos identificados con las letras del alfabeto a fin de evitar el carácter predominantemente jerárquico que suele atribuirse al uso de números.

Resultados. El alumnado masculino ha concluido que percibe haber cometido más trasgresiones de orden instruccional que de carácter convencional durante su escolarización. Esto nos debería poner en alerta a docentes y a los propios discentes. La verificación de estas diferencias es congruente con estudios anteriores del profesorado, donde existe cierta preocupación descontextualizada por parte de los docentes en lo referente a los comportamientos que poseen mayor poder disruptivo en el aula. Por otro lado las mujeres del estudio creen recordar haber cometido menos actos indisciplinados que los hombres (cosa que correspondería con la literatura sobre género y comportamientos de disciplina en el aula).

Discusión y conclusión. Estos resultados ponen de manifiesto que, una vez más, las diferencias entre hombres y mujeres en aspectos de comportamiento se manifiestan claramente. Queda patente que tanto hombres como mujeres recuerdan haber cometido más transgresiones de disciplina instruccional que de indisciplina social durante su etapa escolar. No obstante y como se mantiene en la literatura sobre el tema, son los aspectos sociales los que continúan preocupando más al profesorado y al alumnado, probablemente fruto de sus creencias.

Palabras Clave: Disciplina en el aula, entornos instruccionales, diferencias individuales, género.

Recibido: $29 / 12 / 11$

Aceptación inicial: $\quad$ 05/05/12

Aceptación final: $22 / 06 / 12$ 


\title{
Social and instructional indiscipline in teenagers and uni- versity students: the influence of gender
}

\begin{abstract}
Introduction. There are several studies on gender differences in school. Some have referred in treatment over their pupils by gender, others have referred to the perceptions of teachers and students about school discipline. But there is little research that has taken into account the gender variable in the study of social and instructional behavior. In this paper, we make an approach to this topic.

Method. This research is part of an empirical-analytical quantitative study that compares the data obtained from the application of a questionnaire of 456 subjects, from high school students of Barcelona and Madrid and the Autonomous University of Barcelona (Spain). For this study we developed a tool consisting of a target with concentric circles and a list of disruptive behaviors, to minimize the distortions associated with language, from the graphical representation of a target.

Results. Male students have concluded that perceived transgressions they had committed belong more to instructional order that to conventional. This should put us on alert, both teachers and learners themselves. Verification of these differences is consistent with previous studies of teachers, where there is some concern out of context by teachers regarding the behaviors that have more disruptive power in the classroom. On the other hand women in the study believe to have committed fewer undisciplined acts than men (which correspond to the literature on gender and discipline behaviors in the classroom).

Discussion and Conclusion: These results demonstrate once again that the differences between men and women in aspects of behavior are clearly evident. It is clear that both men and women remember having committed more transgressions of instructional discipline than social indiscipline during their time at school. However, as it is maintained in the literature on the subject, are the social aspects which remain more concerned faculty and students, probably due to their beliefs.
\end{abstract}

Keywords: Classroom discipline, instructional environments, individual differences, gender. 


\section{Introducción}

Los estudios que encontramos en la literatura sobre las diferencias de género en la escuela, tienden a señalar un mayor reconocimiento de los niños y las niñas por igual, dejando atrás antiguos esquemas de dominación, machismo y autoritarismo aplicados en otras épocas. Algunos de estos estudios han contemplado el número de intervenciones del profesor y también las diferencias de trato respecto a los alumnos en función del género (Brullet y Subirats, 1989). Otras revisiones han revelado que se han producido pocos cambios en las últimas tres décadas respecto a las percepciones aplicadas a las niñas (Skelton y Francis, 2003). No obstante, las diferencias de género no representan el cien por cien de las variaciones en el comportamiento del alumnado: los alumnos y las alumnas no se comportan presentando estereotipos de género de manera constante, e incluso hay quien no se comporta nunca de acuerdo a esta pauta.

Por otra parte, los géneros masculino y femenino son elementos de construcción social, constantemente afectados por el poder que impone un tipo de feminidad y de masculinidad a través de un sistema, régimen u orden de sexo/género. Respecto a este punto, existen presiones, a nivel moral e ideológico, hacia un "deber ser" normativo y preferido. Se llega a hablar de contratos implícitos, que consisten en acuerdos y obligaciones desprendidas de normas sociales y morales compartidas en una sociedad, (Francis 2005). Además, el género como herramienta para el análisis social ha sido incorporado de manera masiva en los últimos años. Sin embargo, su adecuada utilización exige una mirada crítica a las formas tradicionales de relación, a cuestionar nuestros valores y creencias, así como a tratar de imaginar un mundo en el que las relaciones sociales están basadas en la equidad.

En ocasiones, los profesores han tendido a considerar que las diferencias de atención no se deben a que los niños y las niñas tienen inquietudes e intereses diversos, sino que se trata de un tema innato vinculado al género del alumno. De hecho, en la literatura a veces se ha generalizado afirmando que los chicos son más inquietos, pero a su vez más inteligentes y que las niñas son calladas, tímidas y que se comportan mejor en clase. Asimismo, se considera que los chicos suelen ser más ruidosos, por lo que desconcentran a sus compañeros en clase, hablando fuerte, haciendo más ruido, y moviéndose más en el aula. Por su parte, las niñas tienen la imagen de ser más dóciles, más tranquilas, no tan sujetas a distracciones exteriores a la escuela, y se acomodan mejor a la disciplina escolar que los niños (Brullet y Subirats, 
1989). Estas diferencias de personalidad pueden repercutir en las calificaciones tanto por la cuantía de materia realmente aprendida, como y más directamente, en virtud de la impresión percibida por el profesor. En este sentido, es indudable señalar que las diferencias entre los sexos marcan la pauta para las conductas socialmente esperadas en cada individuo, pues por ser biológicamente distintos no podemos actuar igual pero sí de forma incluyente (Bernal, 2005).

También es cierto que hasta hace muy poco tiempo no hemos sabido nada de las niñas, ni del mal comportamiento de éstas, ya que eran invisibles en este tipo de estudios. Por el contrario, los niños han sido investigados, tanto en su mal comportamiento como en todo lo que ellos han hecho ya que han sido objetos de numerosos estudios. (Vaello, 2005). Una parte del problema es ocasionado por las relaciones que se establecen entre las niñas y los profesores/as, que muchas veces suelen ser engañosas y ficticias. Davies (1984) por ejemplo, demostró en los datos obtenidos de sus investigaciones que las alumnas que se mostraban impertinentes e indisciplinadas eran las que se sentían resentidas con los profesores/as y les guardaban rencor. Measor y Woods (1984) observaron que el mal comportamiento de las alumnas se asociaba a malos recuerdos que conservaban de anteriores docentes y que hacían extensivo a los actuales. Esto significó que muchos profesores/as, especialmente los varones, tuvieran problemas para encontrar las estrategias apropiadas para mantener la disciplina con las niñas en el aula.

Algunas investigaciones han constatado que los niños tienden a dominar verbalmente las aulas mixtas. Asimismo, muestran que las niñas son más calladas, de manera que suelen quedar en segundo plano (en lo que a conversaciones en el aula se refiere) (Francis, 2000; Younger y Warrington, 1999; Warrington y Younger, 2000). El impacto que esto tiene en los docentes es elevado ya que los profesores recuerdan mejor los nombres y las características personales de los niños, viéndolos como más capaces académicamente hablando, aunque tiene repercusiones en su conducta hacia ellos. Así pues, una parte de la atención que se dedica a los niños en el aula está relacionada con la disciplina.

Aún hoy en día, al preguntar a los adultos cómo creen que actuará una niña, manifiestan esperar que muestre comportamientos femeninos, que sea tímida, callada y educada, en contraste con los comportamientos de los chicos, que se espera que sean más agresivos, que utilicen vocabulario más grosero, con insultos, etc. Si un chico muestra debilidad, o tiene 
comportamientos considerados "femeninos" como ser más dulce, cariñoso, no demasiado ruidoso, etc. puede que se ponga en entredicho su virilidad, siendo un aspecto sumamente nocivo para la configuración de su autoestima (Askew y Ross, 1995). Autores como Epstein (1998) y Francis (2000) argumentan que los niños utilizan el abuso verbal para construir una masculinidad "adecuada".

Algunos estudios (Grebennikov, 2005; Marrero, 2007), resaltan que las niñas, mediante su comportamiento obediente en la escuela, obtienen reconocimiento, mientras que para los chicos, aceptar la normativa escolar les supondría desplazarse al mundo de lo femenino. Las niñas que están en riesgo de graves faltas de disciplina escolar es probable que experimenten dificultades en varios aspectos (académicos, conductuales, emocionales, sociales), y tienden a tener relaciones sociales que apoyan y refuerzan sus problemas de comportamiento (Farmer, Goforth, Leung, Clemmer y Thompson, 2004; Moreno, Martínez y Cervelló, 2009). En la misma línea, Josephs, Markus y Tafarodi (1992) observaron que los hombres se perciben a sí mismos con habilidades racionales, mientras que las mujeres lo hacen con las habilidades emocionales, a pesar de lo cual, es importante remarcar que ambos poseen dichas capacidades.

Igualmente, Fize (2003), en su estudio "las trampas de la enseñanza mixta" refleja que en el mejor rendimiento de las chicas influye su mayor capacidad de adaptación al sistema educativo actual, mientras que a los chicos -sobre todo en ambientes populares- les cuesta más aceptar la disciplina escolar y sufren más el choque entre la cultura adolescente y la cultura académica. Además, la violencia contra las chicas se debe principalmente a un desarreglo general en las relaciones entre los sexos, aunque la enseñanza mixta da ocasión evidentemente a que se manifiesten también en la escuela.

De la misma manera que existen estudios sobre género y cómo este influye en los comportamientos y percepciones de los docentes, otros estudios han abordado cómo perciben profesores y alumnos la disciplina en la clase (Gotzens, Castelló y Badia, 2003), observándose diferencias significativas entre las percepciones de profesores y alumnos respecto a los comportamientos sancionados y de las estrategias punitivas empleadas. Pero pocos han sido los que han puesto su foco de atención en el estudio de género de los alumnos respecto a la indisciplina convencional e instruccional. 
Gotzens, Badia, Castelló y Genovard (2007) realizaron una investigación sobre el estudio comparativo de la gravedad atribuída a las conductas disruptivas en el aula. Los resultados obtenidos destacan, sistemáticamente, que el profesorado muestra mayor preocupación por las transgresiones de orden social general que se dan en el grupo clase, relegando a posiciones de menos importancia las conductas disruptivas de tipo instruccional cuya implicación en los procesos de enseñanza-aprendizaje está rigurosamente comprobada. Por otra parte, en el estudio de Cervelló y Jiménez (2001), se incluyó la disciplina y se manifestó que la percepción de un clima motivador que implicaba a la tarea se relacionaba positivamente, tanto con la percepción de un trato más igualitario por parte del docente a su alumnado cómo con una buena gestión de la disciplina en clase por parte de los discentes. Este trabajo también constató que la percepción de un clima motivador que implicaba al ego se relacionaba con una mayor percepción de discriminación, así como con comportamientos más intensos de indisciplina en el aula.

Llegados a este punto, nos preguntamos si realmente hay tantos problemas de disciplina convencional como para que el profesorado se preocupe, o más bien lo que se da en el aula son conductas de carácter instruccional, que pareciendo ser menos distorsionadoras, son más perjudiciales para el proceso de enseñanza-aprendizaje. También interesa indagar si la literatura desarrollada hasta el momento sobre género (masculino-femenino) y disciplina, guarda relación con las percepciones que los alumnos y alumnas creen tener de ellos mismos. Con el propósito de llevar a cabo los análisis citados, es necesario establecer el concepto de disciplina escolar, así como las diferencias que existen entre disciplina convencional o (social) e instruccional.

\section{Disciplina escolar}

La disciplina escolar es, en la actualidad, una de las principales preocupaciones a la que se enfrenta el profesorado tanto de enseñanza primaria como de secundaria (Benítez, García-Berbén y Fernández, 2009). Pese a ello, la preparación que reciben durante el período de formación docente es casi inexistente, ya que son escasos los centros que introducen dicha temática en el currículum oficial de los futuros enseñantes. Por este motivo, el conocimiento disponible para afrontar dicha temática procede básicamente de sus creencias y sus experiencias y, en consecuencia, es de carácter vivencial y está falto de rigor, tal y cómo se ha podido verificar en otros estudios (Gotzens, Castelló, Genovard y Badia, 2003; Hardman y Smith, 
2003). Asimismo, los problemas de disciplina escolar tienden a estar unidos a los desórdenes de comportamiento disruptivo en la infancia y la adolescencia, que incluyen: dificultades académicas, problemas de comportamiento, dificultades de habilidades sociales, problemas de relación con los compañeros, etc.

En términos generales, es de resaltar la tendencia que sobre este particular se ha encontrado en otros estudios (Dixon y Wilke, 2007; Gotzens, et al., 2007). Los docentes acostumbran a otorgar mayor preponderancia a las infracciones que denominamos de tipo convencional, que a las de tipo instruccional, a pesar de que aun siendo ambas importantes para el orden y funcionamiento del aula, las instruccionales resultan imprescindibles para el cabal desarrollo del proceso de enseñanza-aprendizaje, además de ser las que los profesores pueden gestionar y modificar de forma directa (Gotzens, et al., 2007).

\section{Diferencias entre indisciplina convencional e instruccional}

\section{La indisciplina convencional}

La indisciplina convencional se concibe como el incumplimiento de las normas, reglas y mandatos establecidos por la sociedad. Está vinculada al comportamiento, la conducta y la actitud que asumen tanto el individuo como los grupos sociales ya sea frente a un reto, tarea, problema, necesidad o motivación, con manifestación pública e implicación para la sociedad. La disciplina convencional, es un prototipo con respecto al cual cada miembro de la sociedad se contempla a sí mismo, se compara con los demás, estructura las características de cómo debe proceder y se autoevalúa (Varona, 2008).

Para mantener la disciplina convencional es imprescindible la solidez de los vínculos entre la familia, la escuela y las instituciones sociales encargadas de definir qué es lo que se puede hacer y lo que no se puede hacer, así como las sanciones pertinentes. Este proceso se favorece con la armonía de las relaciones entre los individuos y la sociedad. Si éstas se dañan, se crea un terreno propicio para el surgimiento de comportamientos, conductas o actitudes antitéticas a la disciplina establecida. Es importante que cada individuo conozca los reglamentos disciplinarios y los rasgos que deben tener los miembros de la sociedad para que sean considerados como disciplinados. 
La indisciplina convencional es la negación de la disciplina, su antítesis. Está relacionada con la débil observación del orden establecido por la sociedad y su incumplimiento. Se observa en la falta de correspondencia entre la actuación de un individuo o de grupos sociales y los principios, normas y objetivos de la sociedad en una circunstancia, reto, tarea, problema, necesidad o motivación determinada.

\section{La indisciplina instruccional}

La indisciplina instruccional es la que deriva de los comportamientos disruptivos provocados por el alumno o grupo de alumnos, que no permiten o impiden el buen funcionamiento del aula. Se trata de comportamientos desadaptados porque imposibilitan o dificultan el aprendizaje, y consisten en: hablar sin permiso, interrumpir al profesor, olvidar el material escolar, etc. (Calvo, García y Marrero, 2005).

En el estudio llevado a cabo por la FETE Enseñanza (Federación de Trabajadores de la Enseñanza) sobre los comportamientos disruptivos en las aulas el 27 de enero de 2010, se deduce que los comportamientos de indisciplina que se dan con mayor frecuencia en la relación entre alumnos y profesores son los instruccionales, o sea, aquellos que implican constantes interrupciones de la clase, cómo contestaciones inadecuadas o hablar continuadamente en clase, conductas todas ellas que impiden el normal desarrollo de la tarea docente. Según los datos de este estudio, obtenidos mediante un cuestionario dirigido a 1.300 trabajadores de centros públicos, concertados y privados y entrevistas a directores y profesores de 14 Comunidades Autónomas, se observó que dichos comportamientos se producen con 'mucha frecuencia' según el 34,1\% del profesorado, y 'con cierta frecuencia', es decir, al menos una vez al mes, para el 56,5\% de los docentes. Toda vez que un 37,5\% de los docentes se considera bastante estresado por la interacción con los alumnos. Frente a ellos, un 5\% afirma no sentir ningún grado de estrés en su relación cotidiana con los escolares. En cuestión de género, se aprecia una referencia de percepción significativa. Las mujeres sienten niveles de estrés más elevados que sus compañeros masculinos.

En conclusión, pues, sería interesante comprobar si realmente los estudiantes tienen la misma percepción que los profesores en general, es decir, que el mayor número de incidentes en las escuelas están relacionados con comportamientos de carácter instruccional y también si se observan diferencias de género en este aspecto, tal cómo indica la literatura sobre el tema. 


\section{Objetivos e hipótesis}

El objetivo de este estudio es analizar si la percepción que tienen los estudiantes, tanto de los aspectos relacionados con la disciplina convencional como los relacionados con la disciplina instruccional, difiere entre los hombres y las mujeres. A la vez, deseamos comprobar, si esta percepción se corresponde con la literatura actual sobre el tema, que indica que los alumnos del sexo masculino son los que causan más problemas de comportamiento en el aula.

\section{Método}

\section{Participantes}

Un total de 456 sujetos (165 hombres y 291 mujeres) participaron en el estudio, de los cuáles, 284 estudiantes eran de bachillerato, de centros de secundaria públicos y privados de Barcelona y Madrid, y 172 estudiantes de la licenciatura de Psicología, alumnos de tercer curso, de la Universidad Autónoma de Barcelona (España).

\section{Instrumentos y variables}

El instrumento que se ha utilizado para este estudio ha sido la escala IG de disciplina (Gotzens, Badia, Genovard y Dezcallar, 2010). Como se puede observar en la Figura y Tabla 1, la versión del instrumento utilizado para este estudio consiste en una diana formada por cinco círculos concéntricos y un listado de 11 comportamientos disruptivos identificados con un número (de 1 a 11).

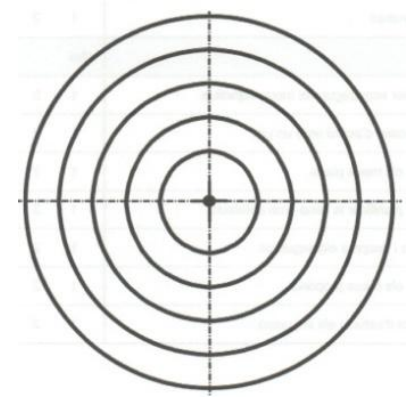

Figura 1. Gráfico para indicar la frecuencia de los comportamientos de indisciplina del alumno.

1. Hablar con los compañeros durante la clase.

2. Destruir material. (fichas de los compañeros, libros, mobiliarios, cristales,......). 
3. No realizar o abandonar las tareas de clase o deberes.

4. Robar y hurtar a los compañeros.

5. Interrumpir al profesor.

6. Desobedecer las normas de clase o hacer caso omiso de las indicaciones de los profesores.

7. Decir "tacos"

8. Acusar a los compañeros.

9. Olvidar materiales escolares a casa

10. Amenazar a los compañeros/profesores.

11. Quejarse siempre de todo

En concreto, las variables utilizadas fueron:

Comportamientos disruptivos: comportamientos no adecuados o poco adecuados de tipo social e instruccional relacionados con los compañeros de clase o el profesor. Se computó la fiabilidad Alfa de Cronback para los 11 ítems de la escala IG (Indisciplina General) obteniéndose un valor $\alpha=.81$. Obsérvese que los compotamientos evaluados, son similares a otros inventarios validados recientemente (De la Fuente, Peralta, Sánchez y Trianes, 2012).

\section{Procedimiento}

En una primera instancia, los sujetos recibían la consigna: "Por favor, sitúa en la siguiente diana los 11 comportamientos que aparecen a continuación. Se trata de distribuirlos según la frecuencia con que recuerdes haberlos cometido a lo largo de $3^{\circ}$ y $4^{\circ}$ de ESO, de manera que aquellos que recuerdes haber cometido con mayor frecuencia los sitúes en los círculos más centrales de la diana, y los que creas haber cometido infrecuentemente los sitúes en los círculos más periféricos. Aquellos comportamientos que recuerdes no haber cometido nunca, debes ubicarlos fuera de la diana”. 
Los alumnos que participaron en el estudio, tenían que ubicar los números correspondientes a cada comportamiento en algún lugar de la diana, teniendo en cuenta que cuanto más hacia el centro se situaran, mayor era la frecuencia con que declaraban haber presentado el comportamiento, y cuanto más alejados del punto central, menor era su frecuencia de presentación. A cada comportamiento se le otorgaron 5 puntos si estaba ubicado en el centro de la diana, 4 en el siguiente círculo y así sucesivamente hasta 0 puntos si se ubicaba fuera del círculo exterior. Así pues, la escala de puntuación tenía un rango efectivo de 0 a 5 puntos. Cabe añadir que los estudiantes colaboraron de forma totalmente voluntaria y que en todo momento se garantizaba el anonimato de los mismos.

Así, tras contactar con los directores de los centros escolares y los profesores de la universidad, se concertó día y hora para la administración de la prueba. A pesar de no establecer límite de tiempo ningún alumno superó los 15 minutos en contestar el cuestionario.

\section{Análisis de datos}

La investigación se enmarca en un diseño cuantitativo empírico-analítico, basada en un estudio de carácter descriptivo con la aplicación de un instrumento creado para este fin. En primera instancia las respuestas fueron sometidas a un análisis factorial para la posible distribución de los comportamientos disruptivos en uno o más grupos homogéneos. Posteriormente se efectuó la prueba de comparación de medias para muestras independientes, tomando como variable independiente, el género, y como variables dependientes, las puntuaciones en indisciplina instruccional e indisciplina convencional.

\section{Resultados}

A partir de unos primeros resultados obtenidos a través de la prueba de análisis factorial nos planteamos mantener todos los ítems o eliminar aquellos que presentaran valores bajos. La solución final adoptada se corresponde con esta última. Así, se eliminaron tres ítems 7, 9 y 11 que presentaban valores de extracción inferiores a .40 (Peterson, 2000). Con los 8 restantes se han extraído dos factores que explican el $60.97 \%$ de la varianza de los datos originales. La Tabla 1 muestra estos valores. 
Tabla 1. \% de la varianza y \% acumulado de los diferentes componentes.

\begin{tabular}{|l|l|l|l|}
\hline & \multicolumn{3}{|c|}{ Autovalores iniciales } \\
\hline Componente & Total & $\begin{array}{l}\text { \% de la } \\
\text { varianza }\end{array}$ & \% acumulado \\
\hline 1 & 3.35 & 41.88 & 41.88 \\
\hline 2 & 1.53 & 19.09 & 60.97 \\
\hline 3 & .69 & 8.55 & 69.52 \\
\hline 4 & .64 & 8.02 & 77.54 \\
\hline 5 & .56 & 7.06 & 84.61 \\
\hline 6 & .49 & 6.07 & 90.68 \\
\hline 7 & .43 & 5.39 & 96.07 \\
\hline 8 & .32 & 3.93 & 100 \\
\hline
\end{tabular}

Un primer factor, que se correspondería con los comportamientos disruptivos de carácter instruccional estaría formado por los ítems 1,3 y 5 ; mientras que un segundo factor de comportamientos disruptivos de carácter convencional o social estaría formado por los ítems 2, 4, 6, 8 y 10. En la siguiente Tabla 2 se muestran las correlaciones entre los diferentes ítems pertenecientes a un mismo factor. En un posterior análisis estadístico se efectuó la prueba de comparación de medias para grupos independientes entre las variables género y tipología de indisciplina.

Tabla 2. Correlaciones entre los diferentes ítems (p<.01)

\begin{tabular}{|c|c|c|c|c|c|c|}
\hline & Ítem3 & Ítem5 & İtem4 & İtem6 & Ítem8 & Ítem10 \\
\hline Ítem1 & \multirow[t]{2}{*}{.36} & .43 & & & & \\
\hline Ítem3 & & .36 & & & & \\
\hline Ítem2 & & & .45 & .43 & .42 & .55 \\
\hline Ítem4 & & & & .25 & .44 & .59 \\
\hline Ítem6 & & & & & .30 & .34 \\
\hline Ítem8 & & & & & & .63 \\
\hline
\end{tabular}

En la Tabla 3 se puede observar como en los dos tipos de indisciplina se observan diferencias significativas entre hombres y mujeres. En concreto, las puntuaciones de los hombres son significativamente superiores que las de las mujeres en los dos casos. 
Tabla 3. Medias y Desviaciones típicas por género y tipo de indisciplina.

\begin{tabular}{|l|l|l|l|l|l|}
\hline & \multicolumn{1}{|c|}{ Género } & \multicolumn{1}{|c|}{$\mathrm{N}$} & \multicolumn{1}{|c|}{ Media } & \multicolumn{1}{c|}{ Desv. Típica } & t \\
\hline \multirow{2}{*}{$\begin{array}{l}\text { Indisciplina } \\
\text { Instruccional }\end{array}$} & Másculino & 165 & 2.65 & $(.94)$ & \multirow{2}{*}{$4.71 *$} \\
\cline { 2 - 5 } & Femenino & 291 & 2.23 & $(.89)$ & \\
\hline $\begin{array}{l}\text { Indisciplina } \\
\text { Convencional }\end{array}$ & Masculino & 165 & .98 & $(.74)$ & $5.57 *$ \\
\cline { 2 - 5 } & Femenino & 291 & .64 & $(.57)$ & \\
\hline \multicolumn{2}{|l|}{$\mathrm{p}<.000$}
\end{tabular}

\section{Discusión y conclusiones}

No se puede hablar de verdadera enseñanza si no se da comunicación, pues debe haber contacto entre docente y discente para que haya ajustes en el proceso educativo. Esta comunicación deberá ser igualitaria tanto para alumnos como para alumnas, de manera que no se dirija la atención más a unos que a otros. La literatura nos ha demostrado que eso no es así y que siguen existiendo diferencias (Bernal, 2005; Brullet y Subirats, 1989; Younger y Warrington, 1999; Warrington y Younger, 2000); y como se ha podido comprobar en el estudio, estas diferencias se reflejan en las respuestas que nos han dado los estudiantes respecto a su comportamiento en el aula.

La falta de empatía o la dificultad para interpretar las expresiones faciales, que con frecuencia se atribuyen al género masculino, tiene mucho que ver con el peor comportamiento de los niños. Por el contrario, las niñas se orientan con más facilidad a la expresión emocional e interpretando las miradas de las personas que les rodean descubren si son queridas, admitidas, admiradas, lo que les permite actuar en consecuencia (Askew y Ross, 1995; Epstein, 1998; Francis, 2000). El profesorado debería dar importancia a este aspecto potenciando en el alumnado estas habilidades.

Nuestro análisis nos lleva a concluir que el alumnado masculino de este estudio parece haber cometido más trasgresiones de orden instruccional que de carácter convencional durante su escolarización, como indicaba nuestra hipótesis inicial, y tal y como se refleja en los trabajos de Gotzens, et al., (2010). En este punto es importante recordar, que tal como hemos observado en estudios ya citados más arriba, las conductas disruptivas de carácter instruccional son las que entorpecen claramente el proceso de enseñanza-aprendizaje. Esto nos debería poner en alerta a docentes y a los propios discentes ya que los resultados contrastan con la idea 
preconcebida de que son los problemas principalmente de carácter social los que más imposibilitan el aprendizaje en la educación formal.

Adicionalmente, hay que resaltar que todos los comportamientos analizados en este estudio se han manifestado de una manera u otra por parte de los estudiantes, siendo fuentes de disrupción en el aula y, por tanto, creando una situación que resulta amenazadora para el proceso de enseñanza-aprendizaje. Este hecho justifica sobradamente la intensa preocupación que el tema de la disciplina escolar entraña hoy en día para la profesión docente y para los educadores en general (Benítez et al., 2009).

La verificación de estas diferencias es congruente con los resultados de estudios previos realizados con docentes, en los que se percibe cierta preocupación "descontextualizada" por parte de los docentes en lo referente a los comportamientos que poseen mayor poder disruptivo en el aula, ya que otorgan especial atención a comportamientos que no son precisamente los más perjudiciales para la gestión del aula, y no obstante la respuesta de los alumnos indica que aquellos comportamientos que se manifiestan más o que ellos dicen recordar haber cometido con más frecuencia e intensidad, son precisamente los de carácter instruccional. Recordemos que en el estudio de (Gotzens, et al., 2007) los profesores no sólo priorizaban la gravedad de la indisciplina convencional, sino que acciones como hablar quedaban relegadas a los últimos puestos del ranking, olvidando el papel fundamental que los procesos atencionales desempeñan en los aprendizajes.

Por otro lado, las mujeres del estudio dicen haber cometido menos actos indisciplinados que sus compañeros masculinos, cosa que resulta coherente con la literatura sobre género y comportamientos de disciplina en el aula. Si bien puntúan más alto en transgresiones de orden instruccional (como hablar en clase, interrumpir al profesor, etc), en cambio puntuan más bajo en transgresiones de orden social general (robar por ejemplo), conductas que se asocian con el caos en el aula y que dicen haber cometido más los hombres de la muestra del presente estudio.

Estos resultados ponen de manifiesto que una vez más las diferencias entre hombres y mujeres en aspectos de comportamiento se manifiestan claramente. Queda patente que tanto hombres como mujeres recuerdan haber cometido más transgresiones de disciplina instruccional que de indisciplina social. No obstante y como se mantiene en la literatura sobre el te- 
ma, son los aspectos sociales los que continúan preocupando más al profesorado e incluso al propio alumnado, probablemente fruto de sus creencias.

Es probable que el profesorado presente diferencias en las interacciones que se producen con los alumnos y alumnas en el aula, resultando favorecidos los alumnos por sus constantes interrupciones en clase. Dichas diferencias pueden llegar a transmitir estereotipos de género, en cuyo caso los comportamientos de las alumnas y de los alumnos tenderán a reforzar la imagen femenina y masculina socialmente dominante (Marrero, 2007). Es posible establecer cierta correspondencia con los resultados del estudio sobre expectativas y comportamiento del profesorado y alumnado en relación con la educación física y el género de Vázquez (1999), en tanto el profesorado sigue manteniendo expectativas siempre favorables a los alumnos. No obstante, en el mismo estudio, el profesorado también considera que el impacto de las estrategias educativas puede ser mayor en las alumnas que en los alumnos.

No debemos olvidar que las intervenciones del docente en el aula juegan un papel importante como trasmisoras de valores, actitudes y comportamientos, constituyendo una parte significativa del comportamiento social y psicológico de los estudiantes, de forma que para promover la percepción de igualdad de trato en los estudiantes resulta fundamental crear un clima motivador y ofrecer ciertas estrategias de comportamiento transmitidas por el docente. Así, las habilidades básicas de interacción social, sonreir, saludar, presentarse, ser amable, cooperar, y un largo etc, deberán estar presentes en el día a día de los alumnos. La pregunta es: ¿debemos plantearnos realmente la necesidad de que ambos sexos desarrollen todas las estrategias y valores por igual? Dejamos abierta esta pregunta para que el lector llegue a sus propias conclusiones.

Sólo resta comentar que somos conscientes de la limitación que supone en el estudio hacer recordar al alumno sus vivencias pasadas, pero a la vez también coincidimos en que éstas contituyen una fuente de estudio privilegiada. La memoria nos permite retener experiencias del pasado y es la expresión de que ha ocurrido un aprendizaje. No obstante, de cara a próximas investigaciones quizás sería interesante ampliar la muestra con estudiantes universitarios de otras facultades, que no pertenezcan sólo a la facultad de psicología. También asumimos la limitación que supone el uso de cuestionarios autodescriptivos, ya que, en ocasiones, dificultan el control de la veracidad y la deseabilidad social. Por esa razón nos plateamos 
en un futuro el uso de la observación directa como técnica preferente para la recogida de datos sobre el comportamiento de indisciplina del alumnado.

Para finalizar, deseamos dejar constancia de nuestro interés en llevar a cabo una evaluación de la disciplina en el aula con alumnos de centros escolares extranjeros, de manera que podamos comparar si el tema del comportamiento de indisciplina está vinculado también a aspectos culturales. 


\section{Agracedimientos}

Este artículo se ha realizado en el marco del Proyecto I+D+i ref. EDU2009-10651, Ministerio de Educación y Ciencia. España.

\section{Referencias}

Askew, S. y Ross, C. (1995). Los chicos no lloran. El sexismo en la educación. Barcelona: Paidós.

Badia, M. (2001). Las percepciones de profesores y alumnos de E.S.O sobre la intervención en el comportamiento disruptivo: un estudio comparativo. Tesis Doctoral. Bellaterra (Barcelona). Universidad Autónoma de Barcelona.

Benítez, J. L., García-Berbén, A.B. y Fernández, M. (2009). Impacto de un curso sobre maltrato entre iguales en el currículum universitario del profesorado. Electronic Journal of Research in Educational Psychology, 7(1), 191-208.

Bernal, R. (2005). Violencia de género en la escuela: sus efectos en la identidad, en la autoestima y en el proyecto de vida. Revista Iberoamericana de Educación, 38, 67-86.

Brullet, C. y Subirats, M. (1989). La transmisión de los géneros en la escuela mixta. Madrid: Visor.

Calvo, P., García, A, Marrero, P. (2005). La Disciplina en contextos educativos. Las Palmas de Gran Canaria. Servicio de Publicaciones de la Universidad de Las Palmas de Gran Canaria.

Cervelló, E. y Jiménez, R. (2001). Un estudio correlacional entre la orientación motivacional, el clima motivacional percibido, la coeducación y los comportamientos de disciplina en las clases de Educación Física. IV Congreso Internacional sobre la enseñanza de la Educación Física y el Deporte Escolar. La Didáctica de la Educación Física (pp. 203-209). Santander: ADEF Cantabria.

Davies, L. (1984). Pupil power: deviance and gender in school. London: Falmer.

De la Fuente, J., Peralta, F.J., Sánchez-Roda, M.D. y Trianes, M.V. (2012). Validation Study of the Questionnaire on School Maladjustmet Problems (QSMP). Psicothema, 24 (2), 330-336.

Dixon, P. y Wilke, R. A. (2007). The Influence of a Teacher Research Experience on Elementary Teachers' Thinking and Instruction. Journal of Elementary Science Education, 19, 25-44. 
Farmer, T. W., Goforth, J. B., Leung, M.-C., Clemmer, J. T. y Thompson, J. H. (2004). School discipline problems in rural African American early adolescents: Characteristics of students with major, minor, and no offenses. Behavioural Disorders, 29 (4), 317-336.

Federación de Trabajadores de la Enseñanza (2010). Los comportamientos disruptivos en las aulas generan altos niveles de estrés a más del $50 \%$ del profesorado. Recuperado el $12 / 12 / 2011$, de

http://gdc.feteugt.es/cuteeditornet/imagenes/2010/Comunicacion/COMprensa270110.pdf.

Fize, M (2003). Les Piéges de la mixité scolaire: Presses de la Reinessance.

Francis, B. (2005). Not/Knowing their place: Girls' classroom behavior. En G. Lloyd (ed.), Problem Girls (pp. 63-75). Londres: Routledge.

Francis, B. (2000). Boys girls and achievement: adressing the classroom issues. Londres: Routledge.

Gotzens, C., Castelló, A., Genovard, C. y Badia, M. (2003). Percepciones de profesores y alumnos de E.S.O. sobre la disciplina en el aula. Psicothema, 15 (3), 362-368.

Gotzens, C., Badia, M., Castelló, A. y Genovard, C. (2007). La gravedad de los problemas de comportamiento en el aula vista por los profesores. Revista Portuguesa de Pedagogía, 41 (1), 103-120.

Gotzens, C., Badia M., Genovard, C. y Dezcallar, M.T. (2010). Estudio comparativo de la gravedad atribuida a las conductas disruptivas en el aula. Electronic Journal of Research in Educational Psychology, 8 (1), 33-58.

Grebennikov, L. (2005). The Normalised Child: A Non-Traditional Psychological Framework. Australian Journal of Early Childhood, 30 (2), 8-14.

Hardman, E. L. y Smith, S. (2003). Analysis of Classroom Discipline-Related Content in Elementary Education Journals. Behavioral Disorders. 28 (2), 173-186.

Josephs, R. A., Markus, H. R. y Tafarodi, R. W. (1992). Gender and self-esteem. Journal of Personality and Social Psychology, 48, 393-399.

Marrero, A. (2007). Todas las escuelas, la escuela. Miradas transmodernas sobre educación, Valencia: Germanía.

Measor, L. y Woods, P. (1984). Changing Schools. Open University Press: Milton Keynes.

Moreno, J.A., Martínez, C. y Cervelló, E. (2009). Relación predictiva entre la percepción del alumnado de las estrategias de disciplina del profesor y la percepción del trato de igualdad-discriminación en las clases de Educación Física. Revista de Educación, 355, 381-403. 
Peterson, R. A. (2000). A Meta-analysis of variance Accounted for and factor loadings in Exploratory Factor Analysis. Marketing Letters, 11, 261-275.

Skelton, C. y Francis, B. (2003). Introduction. En C. Skelton y B. Francis (eds). Boys and Girls in the Primary Classroom. Buckingham: Open University Press.

Vaello, J. (2005). Las habilidades sociales en el aula. Madrid: Santillana.

Vázquez, B. (1999). Expectativas y comportamiento del profesorado y el alumnado en relación con la educación física y el género. Madrid: MEC.

Varona, F. (2008). La Indisciplina social. Sus manifestaciones en la ciudad de Holguín a principio del siglo XXI. Revista Electrónica Ciencias Holguín. 4, 1-12.

Warrington, M. y Younger, M. (2000). The other side of the Gender Gap. Gender and education, 12 (4), 493-508.

Younger, M. y Warrington, M. (1999). The gender gapand classroom interactions: reality and rethoric? British Journal of Sociology of Education, 20 (3), 325-341. 